\section{Efficient Sampling of Band-limited Signals from Sine Wave Crossings}

\author{
J. Selva
}

\begin{abstract}
This correspondence presents an efficient method for reconstructing a band-limited signal in the discrete domain from its crossings with a sine wave. The method makes it possible to design A/D converters that only deliver the crossing timings, which are then used to interpolate the input signal at arbitrary instants. Potentially, it may allow for reductions in power consumption and complexity in these converters. The reconstruction in the discrete domain is based on a recentlyproposed modification of the Lagrange interpolator, which is readily implementable with linear complexity and efficiently, given that it re-uses known schemes for variable fractional-delay (VFD) filters. As a spin-off, the method allows one to perform spectral analysis from sine wave crossings with the complexity of the FFT. Finally, the results in the correspondence are validated in several numerical examples.
\end{abstract}

\section{INTRODUCTION}

The analog-to-discrete (A/D) conversion is the first step for the discrete-time processing of continuous signals. This conversion is fundamentally based on the Sampling Theorem, which states that a band-limited signal can be recovered from its regularly-spaced samples taken at least at twice the Nyquist rate. However, some authors early noticed that this recovery is also possible from the signal's zeros, or from its crossings with another signal like a sine wave, [1]-[4]. This is due to the fact that a band-limited signal is an entire function of exponential type, for which there is a factorization in terms of its roots akin to that of conventional polynomials, (Hadamard's factorization theorem [5, chapter 2]). A consequence of this is that it would be possible, in principle, to design $A / D$ converters in which the sample quantization is substituted by a zero crossing detector and an accurate timing, [6]. This new procedure would eliminate the need to quantize any signal samples, so decreasing the complexity and power consumption of $\mathrm{A} / \mathrm{D}$ converters, provided there is an accurate timing available. Besides, it would mainly re-use existing technologies, given that zero crossing detection is implicit in many existing systems. This last point can be readily seen in the current trend in A/D converter design, in which the sample amplitudes are turned into zero crossings, which can then be accurately detected with low-power consumption, [7], [8].

The main obstacle for this alternative procedure is how the signal should be reconstructed or processed in the discrete domain, since Hadamard's factorization theorem does not directly lead to efficient implementations, due to its slow

Copyright (c) 2010 IEEE. Personal use of this material is permitted. However, permission to use this material for any other purposes must be obtained from the IEEE by sending a request to pubs-permissions@ieee.org. The author is with the Dept. of Physics, Systems Engineering and Signal Theory (DFISTS), University of Alicante, P.O.Box 99, E-03080 Alicante, Spain (e-mail: jesus.selva@ua.es). This work has been supported by the Spanish Ministry of Education and Science (MEC), Generalitat Valenciana $(\mathrm{GV})$, and by the University of Alicante (UA) under the following projects/programmes: TEC2005-06863-C02-02, HA2007-075 and "Ramón y Cajal" (MEC); ACOMP07-087 and GV07/214 (GV); and GRE074P (UA). convergence rate. Here, the usual approach in the literature consists in approximating the signal in a finite interval using a trigonometric polynomial, [6], [9], [10]. But then the interpolation error decreases only as $\mathrm{O}(1 / N)$, while the complexity per interpolated value is $\mathrm{O}(N)$, where $N$ is the number of crossings inside the interval. So, in this approach it is necessary to employ a large $N$ to ensure an acceptable accuracy, with the associated high complexity.

The purpose of this correspondence is to present a method for overcoming this obstacle, that makes it possible to reconstruct the bandlimited signal from its sine wave crossings efficiently. The method is based on viewing the reconstruction as a problem of interpolation from nonuniform samples, to which the efficient technique in [11] is applied. Relative to the state of the art, it has several advantages:

- The complexity is reduced significantly. If in the approach in [6], [9], [10], a complexity $\mathrm{O}(N)$ (per interpolated value) gives an interpolation error $\mathrm{O}(1 / N)$, with the proposed method a complexity $\mathrm{O}(N)$ gives an error $\mathrm{O}\left(\mathrm{e}^{-\pi(1-B T) N}\right)$, where $B$ is the signal's two-sided bandwidth and $T$ is the average crossing separation. In practice this means that "any" accuracy can be achieved with a small $N$.

- The method is based on the evaluation of a fixed smooth function and on the Lagrange interpolator. Besides, it can be evaluated with cost $\mathrm{O}(N)$ per interpolated value, and can be implemented by re-using efficient designs for variable fractional-delay (VFD) filters, [11, Sec. IV].

- As a spin-off, the method permits one to perform spectral analysis from sine wave crossings with complexity $\mathrm{O}(N \log N)$, while the usual method has complexity $\mathrm{O}\left(N^{2}\right)$, [6, Sec. IV].

The correspondence has been organized as follows. The next section reviews the state of the art and presents the problem formulation. In it, it is shown that the reconstruction from sine wave crossings can be turned into an interpolation problem from nonuniform samples. Then, this last problem is addressed in Sec. III where the solution adopted is that in the recent reference [11], based on the Lagrange interpolator. The main result is a simple interpolation formula for reconstructing the signal from its sine wave crossings. Then, Sec. IV addresses the problem of analyzing the spectrum from sine wave crossings in the light of the formula in Sec. III It turns out that this formula makes it possible to reduce the complexity from the usual $\mathrm{O}\left(N^{2}\right)$ order to order $\mathrm{O}(N \log N)$. Finally, Sec. $\mathrm{V}$ validates the results in the correspondence through a numerical example.

\section{STATE OF THE ART AND PROBLEM FORMULATION}

The usual representation for a real finite-energy signal $\mathrm{s}(t)$ with spectrum inside $[-B / 2, B / 2]$ is given by the Sampling Theorem. If the samples $\mathrm{s}(n / B)$ are known, this theorem states that $\mathrm{s}(t)$ can be perfectly reconstructed using the series

$$
\mathrm{s}(n / B+u)=\sum_{p=-\infty}^{\infty} \mathrm{s}((n-p) / B) \operatorname{sinc}(p+B u),
$$


where $n$ is an integer and $u$ is any time shift following $-1 /(2 B) \leq u<1 /(2 B)$. This series is the basis of most processing algorithms for continuous signals in the discrete domain. Several authors [1], [2], [4] soon noticed that $s(t)$ can alternatively be viewed as a polynomial of infinite degree, which can be described in terms of its roots. The reason why is that a band-limited signal can be regarded as an analytic function over the whole complex plane, if the $t$ variable is allowed to take complex values. Besides, this kind of function is bounded on the real axis (real $t$ ), and its maximum growth rate is that of $\mathrm{e}^{\pi B|t|}$ along the imaginary axis. It can be shown that this kind of signal admits the representation

$$
\mathrm{s}(t)=K \lim _{R \rightarrow \infty} \prod_{\left|\tau_{p}\right| \leq R}^{\prime}\left(1-t / \tau_{p}\right)
$$

where $K$ is a real constant, and the $\tau_{p}$ are complex roots which appear in conjugate pairs, $\tau_{p} \neq 0$, and may not be distinct, [12, Theorem VI]. The prime (') means that the factor should be replaced with $t$ if $t_{p}=0$ 1. Eq. (2) is the explicit root factorization of $\mathrm{s}(t)$ as infinite-degree polynomial, and from it it is obvious that the roots $\tau_{p}$ determine the signal except for the scale factor $K$. The root density in any circle $|t|<\tau$ follows the same rule as the sample density in (1), i.e, the circle $|t|<\tau$ contains either $2 \tau B$ samples in (1) or $2 \tau B$ roots in (2) asymptotically.

The factorization in Eq. (2) suggested the possibility of performing the usual processing of continuous signals in the discrete domain using the zeros of the signal instead of its samples. In such approach, the analog-to-discrete (A/D) conversion would consist in acquiring the signal's zeros, and the discrete-to-analog (D/A) conversion would be based on the evaluation of a formula like (2). However, it was soon realized that there were two main problems, [4], [6]. The first was how the roots should be located efficiently in the A/D conversion, since they may have a non-null imaginary part. And the second was how the infinite product in (2) should be approximated so as to implement the D/A conversion, since in practice only a finite sequence of roots $\tau_{p}$ is known, and (2) converges slowly.

A solution for the first problem was readily found [4, Sec $\mathrm{V}]$, and consisted in subtracting a sinusoidal $A_{s} \sin (\pi B t)$ to $\mathrm{s}(t)$, where

$$
A_{s}=\sup _{t \text { real }}|\mathrm{s}(t)|
$$

This simple procedure actually solved the problem of locating the roots, because the subtraction of this sine wave "moves" all roots to the real axis, due to a theorem of Duffin and Schaeffer, [13]. Specifically, $\mathrm{s}(t)-A_{s} \sin (\pi B t)$ can only have zeros on the real axis, and each of them can be viewed as a zero of $A \sin (\pi B t)$, which has been shifted by at most $1 /(2 B)$. So, in notation, all zeros of $\mathrm{s}(t)-A_{s} \sin (\pi B t)$ have the form

\footnotetext{
${ }^{1}$ The factorization in 2] is due to Titmarsch [12, Theorem VI], and is a refinement of Hadamard's factorization theorem [5, chapter 2] for $\mathrm{s}(t)$ bounded on the real axis. Note that there is a bug in Eq. [4] of [11]. This last equation is Hadamard's factorization and its correct form is
}

$$
\phi(t) \equiv A \mathrm{e}^{-t \sum_{n} 1 / t_{n}} \prod_{n=-\infty}^{\infty}\left(1-t / t_{n}\right) \mathrm{e}^{t / t_{n}} .
$$

$n / B+\delta_{n}$, where $n$ is an integer and $\left|\delta_{n}\right| \leq 1 /(2 B)$, and two consecutive zeros may overlap only at instants $n / B+1 /(2 B)$. Using this description, the factorization in Eq. (2) for $\mathrm{s}(t)-$ $A_{s} \sin (\pi B t)$ is

$$
\mathrm{s}(t)-A_{s} \sin (\pi B t)=K^{\prime}\left(t_{0}-t\right) \prod_{k=1}^{\infty}\left(1-\frac{t}{t_{-k}}\right)\left(1-\frac{t}{t_{k}}\right)
$$

where $K^{\prime}$ is a real constant and

$$
t_{n} \equiv n / B+\delta_{n} .
$$

From Eq. (4), the basic design for the desired A/D converter was clear. First an oscillator would be used to generate the wave $A_{s} \sin (\pi t / B)$, which would then be subtracted from $\mathrm{s}(t)$. Afterward, the zero crossings would be detected using a gate, and the converter output would be the sequence of shifts $\delta_{n}$ in Eq. (5).

As to the second problem, the usual solution to date consists in approximating the signal using a trigonometric polynomial, [6]. In short, if the finite sequence of roots $t_{n}, t_{n+1}, \ldots, t_{n+M-1}$ is known, then $\mathrm{s}(t)-A_{s} \sin (\pi B t)$ is interpolated using a trigonometric polynomial of order $M$ which is zero at $t_{n+m}, 0 \leq m<M$. However, this solution is not satisfactory since its accuracy is poor even for a large number of roots. This complexity issue is the main obstacle for achieving efficient implementations.

The purpose of this correspondence is to provide an efficient solution to this second problem. The key point is to realize that approximating $\mathrm{s}(t)$ from the roots $t_{n}$ of $\mathrm{s}(t)-A_{s} \sin (\pi B t)$ is the same as approximating $\mathrm{s}(t)$ from its value at these instants, since $\mathrm{s}\left(t_{n}\right)=A_{s} \sin \left(\pi B t_{n}\right)$. So, this is actually a problem of interpolation from nonuniform samples.

\section{PROposed SOLUTION}

Reference [11] presents an efficient and accurate interpolator for band-limited signals from nonuniform samples, applicable to signals of the same type as $\mathrm{s}(t)$, (bandwidth $B$, supremum amplitude $A_{s}$ ). Consider one such signal $\mathrm{z}(t)$ and assume it is known at a set of instants $\tau_{p}$ of the form

$$
\tau_{p} \equiv p T+\eta_{p},-P \leq p \leq P,
$$

where the period $T$ and the shifts $\eta_{p}$ follow $B T<1$ and $\eta_{p}<T / 2$ (strict inequalities). The approach in [11] consists in applying the Lagrange interpolator to the product $\mathrm{z}(t) \gamma(t)$, where $\gamma(t)$ is a fixed function, and then solving for the value of $\mathrm{z}(t)$. The interpolator's formula is

$$
\mathrm{z}(t) \approx \frac{1}{\gamma(t)} \sum_{p=-P}^{P} \mathrm{z}\left(\tau_{p}\right) \gamma\left(\tau_{p}\right) \frac{\mathrm{L}(t)}{\mathrm{L}^{\prime}\left(\tau_{p}\right)\left(t-\tau_{p}\right)},
$$

where

$$
\mathrm{L}(t) \equiv \prod_{p=-P}^{P} t-\tau_{p}
$$

and $\gamma(t)$ is given in Ap. I and only depends on $B, T$ and $P$. Usually, Eq. (7) is applied for $|t| \leq T / 2$, though wider ranges are allowed. In [11], it was shown that the accuracy of (7) increases exponentially with $P$. So, in practice a small $P$ is enough to obtain high accuracy. 
To assess the accuracy of (7), assume $B T=0.7$ and $|\mathrm{z}(t)| \leq 1$. Then, following the analysis in [11, Sec. III], the interpolation error of (7) for the function $\gamma(t)$ specified in Ap. 【is well fitted by

$$
\begin{aligned}
\epsilon(\mathrm{dB}) & \approx 4.12106+66.6044 \delta-9.35838 \delta^{2} \\
& -8.30873 P+3.13419 \delta P-0.125803 \delta^{2} P,
\end{aligned}
$$

where $\delta$ is a bound on the deviations of the instants $\tau_{p}$ from a uniform grid with spacing $T$ and $\delta<T / 2$. So, if $\delta=T / 4$ the value $P=10$ gives an interpolation error below $\epsilon=-55 \mathrm{~dB}$, and $P=16$ gives an error below $\epsilon=-100 \mathrm{~dB}$. Any practical accuracy can be obtained by slightly increasing $P$ for fixed $\delta$. (For a detailed analysis, see the previous reference.)

Coming back to the problem in the previous section, the sine wave crossings are equivalent to nonuniform samples of a form similar to that in (7). Specifically, the crossing instants in (5) have a maximum deviation from the uniform grid, $\left|\delta_{n}\right| \leq$ $1 /(2 B)$, and the sample values are given by the sine wave, since

$$
\mathrm{s}\left(t_{n}\right)=A_{s} \sin \left(\pi B t_{n}\right)=A_{s}(-1)^{n} \sin \left(\pi B \delta_{n}\right) .
$$

However Eq. (7) is not applicable to $\mathrm{s}(t)$ since the regular grid in (5) matches exactly the Nyquist rate and it may be $\left|\delta_{n}\right|=1 /(2 B)$, while in (6) there is some sampling inefficiency, $B T<1$, and it is always $\left|\eta_{p}\right|<T / 2$. These latter conditions can be easily imposed on the sampling scheme by slightly increasing the amplitude and frequency of the sine wave. So if $A_{s} \sin (\pi B t)$ in (4) is replaced by another sine wave $A \sin (\pi t / T)$, with amplitude $A>A_{s}$ and semi-period fulfilling $T>0$ and $B T<1$, the theorem of Duffin and Schaeffer in the previous section ensures that the only zeros of the signal

$$
\mathrm{s}(t)-A \sin (\pi t / T)
$$

are simple and occur at positions

$$
t_{n}=n T+\delta_{n}
$$

for integer $n$. Besides, the shifts $\delta_{n}$ now follow the strict inequality $\left|\delta_{n}\right| \leq \delta<T / 2$, where

$$
\delta \equiv(T / \pi) \arcsin \left(A_{s} / A\right),
$$

since there can be no zero crossings whenever $A|\sin (\pi t / T)|>A_{s}$.

To check these conditions numerically, consider the BPSK signal in Fig. 1. This signal was generated by modulating a raised cosine pulse of roll-off 0.2 and bandwidth $B=0.7 / T$ with a sequence of random amplitudes \pm 1 . Then the signal's peak amplitude was scaled to 1. Fig. 2] shows the zone marked with a rectangle in Fig. 11 together with the sine wave $A \sin (\pi t / T)$. The conditions in (12) and (13) mean that the crossings with the sine wave can only take place inside the shaded rectangles, and there is exactly one in each of them, as can be seen in this example.

Let us derive the final interpolation formula. Assume $\mathrm{s}(t)$ must be interpolated at an arbitrary $t$ from the crossings with $A \sin (\pi t / T),\left(A>A_{s}, B T<1\right)$. Any $t$ can be written uniquely in the form $t=n T+u$ with integer $n$ and

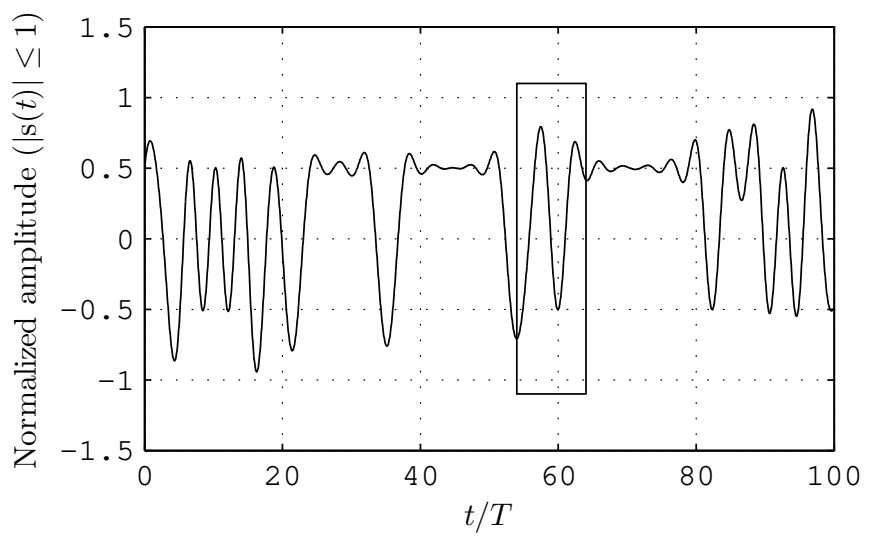

Fig. 1. Random BPSK signal with bandwidth $B=0.7 / T$ and peak amplitude 1 . The symbols used to generate this signal were random \pm 1 values, and the modulating pulse was a raised cosine with roll-off factor 0.2. The signal's peak amplitude was scaled to one.

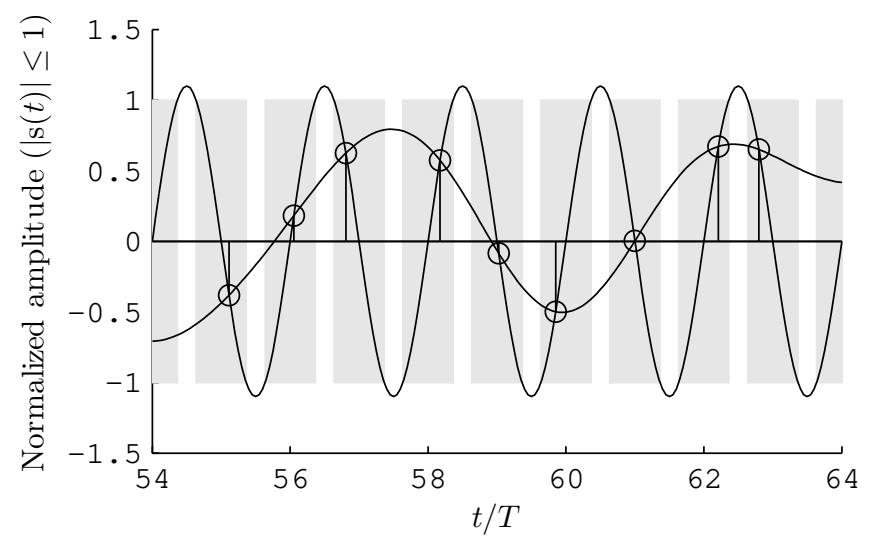

Fig. 2. Short piece of the BPSK signal in Fig. 1 overlapped with the sine wave $A \sin (\pi t / T)$, with $A=1.1$. The stems indicate the positions of the crossings with the sine wave. These can only take place inside the rectangles, and there is exactly one in each of them.

$-T / 2 \leq u<T / 2$. Besides the signal $\mathrm{z}(u)=\mathrm{s}(n T+u)$ with time variable $u$ is of the same type as $\mathrm{s}(t)$, since a time shift affects neither the bandwidth nor the supremum amplitude. So (7) is valid on $\mathrm{z}(u)$ with instants $\tau_{p}=p T+\delta_{n+p}$ and values $\mathrm{z}\left(p T+\delta_{n+p}\right)=A(-1)^{n+p} \sin \left(\pi \delta_{n+p} / T\right)$, i.e, it is

$$
\begin{aligned}
& \mathrm{s}(n T+u) \approx \frac{1}{\gamma(u)} . \\
& \sum_{p=-P}^{P} \frac{A(-1)^{n+p} \sin \left(\pi \delta_{n+p} / T\right) \gamma\left(p T+\delta_{n+p}\right) \mathrm{L}_{n}(u)}{\mathrm{L}_{n}^{\prime}\left(p T+\delta_{n+p}\right)\left(u-p T-\delta_{n+p}\right)} \\
& =\frac{A(-1)^{n}}{\gamma(u)} . \\
& \sum_{p=-P}^{P} \frac{(-1)^{p} \sin \left(\pi \delta_{n+p} / T\right) \gamma\left(p T+\delta_{n+p}\right) \mathrm{L}_{n}(u)}{\mathrm{L}_{n}^{\prime}\left(p T+\delta_{n+p}\right)\left(u-p T-\delta_{n+p}\right)}
\end{aligned}
$$

where

$$
\mathrm{L}_{n}(u) \equiv \prod_{p=-P}^{P} u-p T-\delta_{n+p} .
$$

Eq. (14) is the final interpolation formula. 


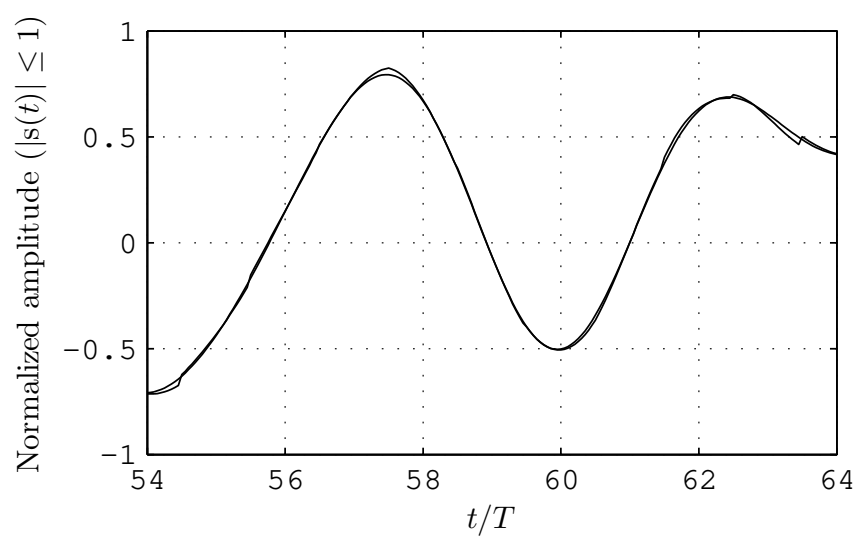

(a) $P=2$

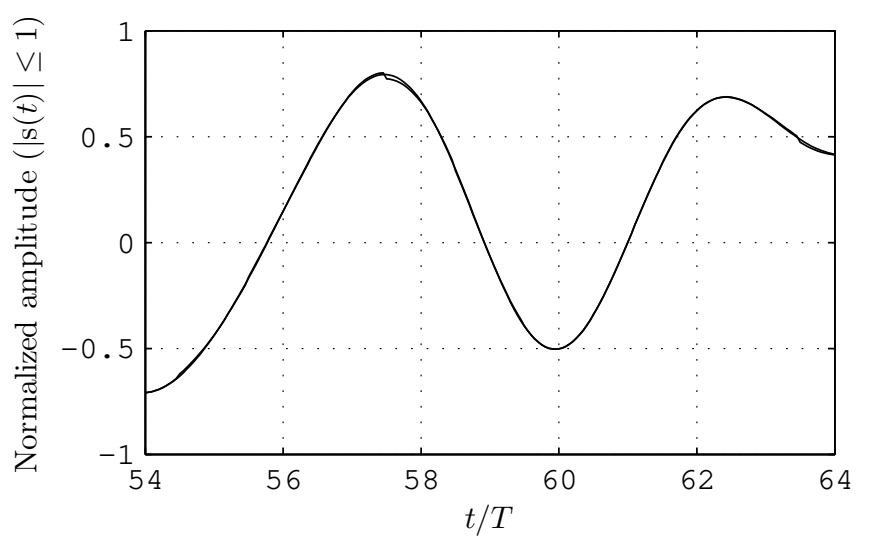

(b) $P=3$

Fig. 3. Sample BPSK signal in 2 and interpolated signal using 14 for $P=2,3$.

For the example in Fig. 2, Fig. 3(a) shows the signal and its interpolated version using (14) for $P=2$. The discontinuities in the interpolated signal take place at $t=n T+T / 2$, integer $n$, because the set of crossings used is different for each $n$. Notice however that just five crossings $(P=2)$ give a good accuracy. Fig. 3(b) shows the same comparison but for $P=3$, (seven crossings). The difference between both signals is much smaller than in Fig. 3(a), For $P>3$ the error becomes too small to be represented this way. For an error analysis see Sec. $\mathrm{V}$

The formula in Eq. (14) yields discrete-time processing methods for delivering samples of $s(t)$ with any spacing, simply by assigning proper values to $n$ and $u$. The simplest case is for spacing $T$, simply by setting $u=0$. For a generic grid of instants $n_{1} T_{1}$ with $T_{1}>0$ and integer $n_{1}$, the grid sample $\mathrm{s}\left(n_{1} T_{1}\right)$ is obtained from (14), simply by setting $n$ and $u$ equal to the modulo- $T$ decomposition of $n_{1} T_{1}$ in (14), i.e,

$$
n=\left\lfloor n_{1} T_{1} / T+1 / 2\right\rfloor \text { and } u=n_{1} T_{1}-n T .
$$

As to the efficient implementation of Eq. (14), it was shown in $[11$, Sec. IV] that it can be evaluated with cost just $\mathrm{O}(N)$. See also the numerical examples in [11, Sec. V].

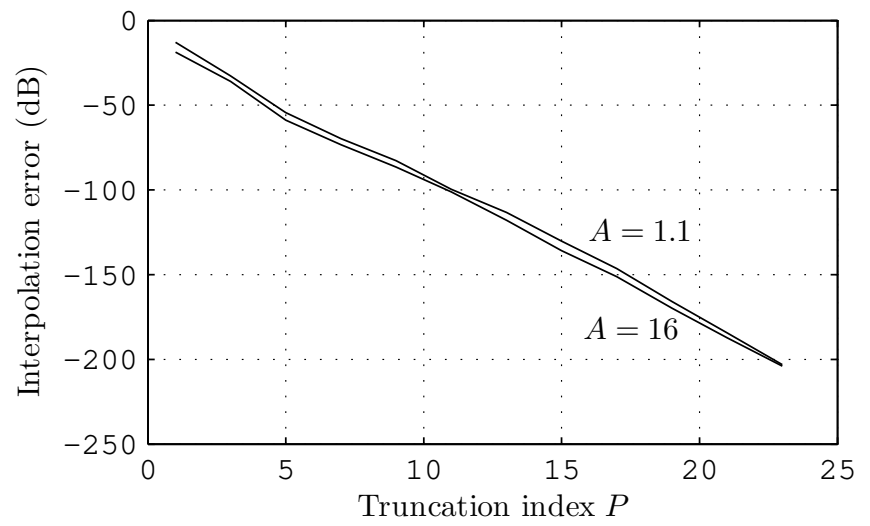

Fig. 4. Error in the interpolation of $\mathrm{s}(t)$ from its sine wave crossings versus index $P$. The error norm is the supremum $\sup _{t \in I}|\mathrm{~s}(t)-\hat{\mathrm{s}}(t)|$ where $\hat{\mathrm{s}}(t)$ is the interpolated signal.

\section{SPECTRAL ESTIMATION FROM SINE WAVE CROSSINGS}

The formula in (14) makes it possible to interpolate the input signal at any instant from its crossings with the sine wave, and the cost of this operation is just $\mathrm{O}(-\log \epsilon)$, where $\epsilon$ is a bound on the interpolation error. This is because the error of (14) decreases exponentially with trend $\mathrm{e}^{-\pi(1-B T) P}$. So, if $\epsilon$ is set below the working numerical error, Eq. (14) allows one to obtain one sample of $\mathrm{s}(t)$ with a small and constant computational cost. Therefore, the cost of computing $N$ samples in a regular grid with arbitrary spacing $T_{1}$ is $\mathrm{O}(N)$. Once these samples are available, the situation is the usual one in which the signal's spectrum is estimated from regularly spaced samples, and any of the well-known techniques in spectral analysis becomes applicable, [14]. Since these techniques are based on the FFT whose complexity is $\mathrm{O}(N \log N)$, it is clear that the total complexity is also $\mathrm{O}(N \log N)$. A numerical example is presented in the next section.

\section{NUMERICAL EXAMPLES}

\section{A. Sampling of a BPSK signal}

To validate the results in a specific example, a BPSK signal $\mathrm{s}(t)$ was generated with the following parameters,

- Modulating pulse: raised cosine with roll-off 0.2.

- Random amplitudes equal to \pm 1 .

- Total two-sided bandwidth $B=0.7 / T$.

- Time interval $I=[0,(N-1) T]$ with $N=1024$.

Then several numerical experiments were conducted.

The first experiment consisted in interpolating $\mathrm{s}(t)$ in $I$ from its sine wave crossings. The result is shown in Fig. 4 for $A=$ 1.1 and $A=16$, where the error norm is the maximum over $I$, that is, if $\hat{\mathrm{s}}(t)$ is the interpolated signal, then the ordinate in this figure is

$$
\sup _{t \in I}|\mathrm{~s}(t)-\hat{\mathrm{s}}(t)|
$$

Notice that this error decreases exponentially with $P$. Besides, the values of $\delta$ for $A=1.1$ and $A=16$ are $0.36 T$ and $0.02 T$, respectively, but the error is roughly the same in both cases. 


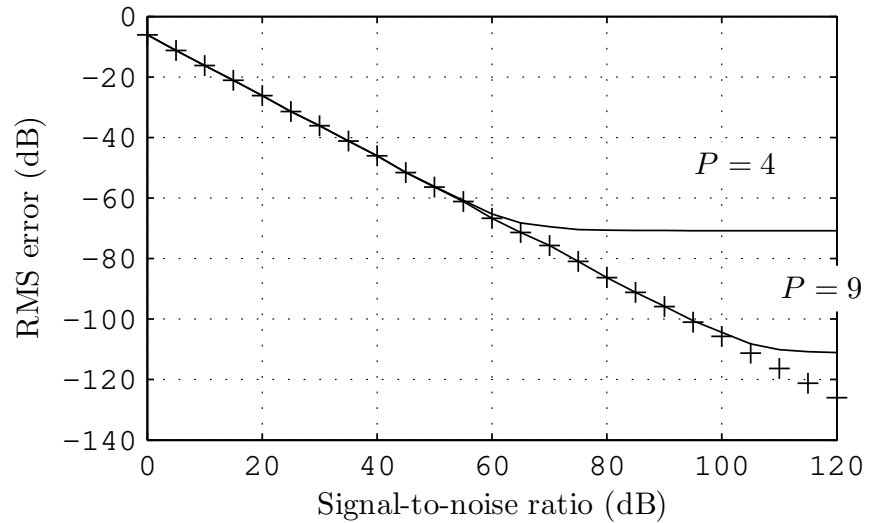

Fig. 5. RMS error versus the SNR in the interpolation of the samples $\mathrm{s}(n T)$ from the sine wave crossings of the noisy realization $\mathrm{s}(t)+\mathrm{w}(t)$.

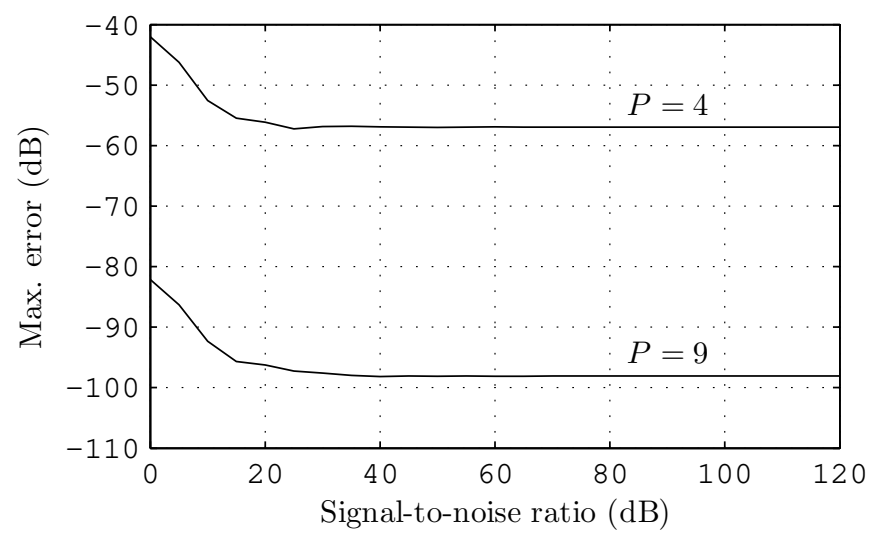

Fig. 6. Maximum difference between the samples $\mathrm{s}(n T)+\mathrm{w}(n T)$ and their interpolated values from sine wave crossings versus the SNR. The error norm if that defined in (19).

So, the fact that the sampling instants may differ from the grid $n T$ (integer $n$ ) has a minimal effect on the performance.

In the second experiment, a white noise process $\mathrm{w}(t)$ of bandwidth $B$ was added to $\mathrm{s}(t)$. Then, $\mathrm{s}(t)+\mathrm{w}(t)$ was sampled at instants $n T$ (integer $n$ ) in $I$, and these samples were also interpolated from the sine wave crossings of $\mathrm{s}(t)+\mathrm{w}(t)$ for $A=3$ and $P=4$ and 9 . Fig. 5 shows the resulting root-meansquare (RMS) error. The crosses $(+)$ indicate the deviation of samples $\mathrm{s}(n T)+\mathrm{w}(n T)$. The other two curves are the RMS errors for $P=4$ and $P=9$, given by

$$
\left(\frac{1}{N} \sum_{n T \in I}\left|\mathrm{~s}(n T)-\hat{\mathrm{s}}_{1}(n T)\right|^{2}\right)^{1 / 2},
$$

where $\mathrm{s}_{1}(t)$ is the value interpolated from the sine wave crossings of the noisy signal $\mathrm{s}(t)+\mathrm{w}(t)$. The curve for either value of $P$ overlaps the sample deviation up to an SNR threshold which is fixed by the specific value of $P$. So, below this threshold, the performance is the same if either the signal is directly sampled, or if it is interpolated from its sine wave crossings. The threshold can be fixed to an SNR as large as desired, simply by slightly increasing $P$, due to the exponential dependence of the interpolation error on $P$.

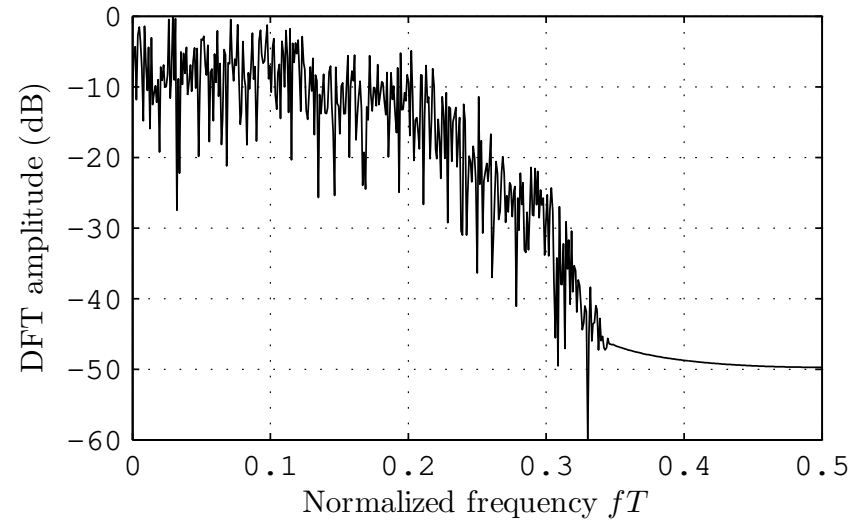

Fig. 7. Amplitude spectrum of sequence $\mathrm{s}(n T)+\mathrm{w}(n T)$ for $n T$ in $I$. The spectrum's maximum value has been normalized to one.

\section{B. Spectral analysis}

As to the spectral analysis, it is worth comparing the conventional procedure from uniform samples, with the one proposed in this correspondence from sine wave crossings. In the conventional procedure, the samples $\mathrm{s}(n T)+\mathrm{w}(n T)$ would be delivered by an A/D converter, and then any of the existing spectral analysis methods would be applied to these data, [14]. And in the proposed procedure, the A/D converter would deliver the sine wave crossing timings $\delta_{n}$, then the uniform samples $\mathrm{s}_{1}(n T)$ would be computed using Eq. (14), and finally the spectral analysis would be the same as in the conventional procedure, i.e, it would be performed on the samples $\mathrm{s}_{1}(n T)$ instead of $\mathrm{s}(n T)+\mathrm{w}(n T)$. The fact is that the result of both procedures would be the same up to the numerical accuracy in use. This can be readily seen in Fig. 6, in which the error measure is the maximum difference between $\mathrm{s}(n T)+\mathrm{w}(n T)$ and $\mathrm{s}_{1}(n T)$,

$$
\sup _{n T \in I}\left|\mathrm{~s}(n T)+\mathrm{w}(n T)-\mathrm{s}_{1}(n T)\right| .
$$

This coincidence is due to the fact that the interpolator in 14 is also reconstructing the noise realization $\mathrm{w}(t)$, since it is also a signal with bandwidth $B$. Fig. (7) shows the amplitude spectrum of the sequence $\mathrm{s}(n T)+\mathrm{w}(n T)$, where the maximum has been normalized to $0 \mathrm{~dB}$. If this spectrum were computed from the sine wave crossings, the it would differ from that if Fig. 7 by the amplitude given in Fig. 8

\section{CONCLUSions}

A method has been presented that makes it possible to recover a band-limited signal from its crossings with a sine wave. It allows one to design A/D converters which only deliver the timing of the sine wave crossings, so allowing for a smaller complexity and power consumption in the converter. The method is based on viewing the problem as one of interpolation from nonuniform samples, to which a recent efficient technique is applied. This technique is based on the Lagrange interpolator and allows for efficient implementations based on current designs of VFD filters. As a spin-off, the method permits one to perform spectral analysis from the sine 


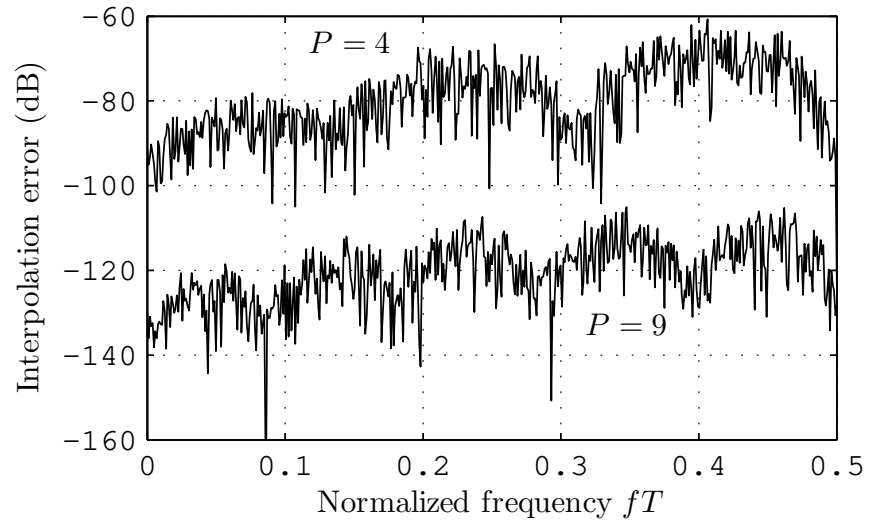

Fig. 8. Amplitude of the difference between the spectrum of $\mathrm{s}(n T)+\mathrm{w}(n T)$ and that of $\mathrm{s}_{1}(n T)$.

wave crossings with the complexity of the FFT. The method has been validated in several numerical examples.

\section{APPENDIX I}

\section{WEIGHT FUNCTION FOR THE LAGRANGE INTERPOLATOR}

Following [11], it is first necessary to define a band-limited window function $\mathrm{w}(t)$ that approximately selects a finite time range. This function is the inverse Fourier transform of the Kaiser-Bessel window,

$$
\mathrm{w}(t) \equiv \frac{\operatorname{sinc}\left(B_{w} \sqrt{t^{2}-T_{w}^{2}}\right)}{\operatorname{sinc}\left(j B_{w} T_{w}\right)},
$$

where

$$
B_{w} \equiv 1 / T-B \text { and } T_{w}=P T .
$$

Note that in (20) the argument of the sinc functions may be pure imaginary. In this case, the sinc can be evaluated from the hyperbolic sine since, for real $a$, it is

$$
\operatorname{sinc}(j a)=\frac{\sin (j \pi a)}{j \pi a}=\frac{\mathrm{e}^{-\pi a}-\mathrm{e}^{\pi a}}{(2 j)(j \pi a)}=\frac{\sinh (\pi a)}{\pi a} .
$$

The weight function $\gamma(t)$ in Sec. IIII is then given by

$$
\gamma(t) \equiv \frac{(-1)^{P}}{(P !)^{2}} \frac{\mathrm{w}(t) \mathrm{L}_{o}(t)}{\sin (\pi t / T)}
$$

where $\mathrm{L}_{o}(t)$ is the Lagrange kernel for the instants $p T,|p| \leq$ $P$,

$$
\mathrm{L}_{o}(t) \equiv \prod_{p=-P}^{P} t-p T .
$$

\section{REFERENCES}

[1] H. B. Voelcker and A. G. Requicha, "Clipping and signal determinism: two algorithms requiring validation," IEEE Transactions on Communications, vol. 21, no. 6, pp. 738-744, 1973.

[2] H. B. Voelcker and A. G. Requicha, "Band-limited real-zero signals," IEEE Transactions on Communications, vol. 21, no. 8, pp. 933-936, 1973.

[3] I. Bar-David, "An implicit sampling theorem for bounded band-limited functions," Information and Control, vol. 24, pp. 36-44, 1974.

[4] A. G. A. Requicha, "The zeros of entire functions: Theory and engineering applications," Proceedings of the IEEE, vol. 68, no. 3, pp. 308-328, Mar 1980.

[5] R. P. Jr. Boas, Entire functions, Acacemic Press, 1954.
[6] S. M. Kay and R. Sudhaker, "A zero-crossing based spectrum analyzer," IEEE Transactions on Acoustics, Speech, and Signal Processing, vol. ASSP-34, no. 1, pp. 96-104, Jan 1986.

[7] H. Lee, L. Brooks, and C. G. Sodini, "Zero-crossing-based ultra-lowpower A/D converters," Proceedings of the IEEE, vol. 98, no. 2, pp. 315-332, Feb 2010.

[8] H. Lee and C. G. Sodini, "Analog-to-digital converters: digitizing the analog world," Proceedings of the IEEE, vol. 96, no. 2, pp. 323-334, Feb 2007.

[9] T. V. Sreenivas and R. J. Niederjohn, "Zero-based spectral analysis and SVD spectral analysis for formant frequency estimation in noise," IEEE Transactions on Signal Processing, vol. 40, no. 2, pp. 282-293, Feb 1992.

[10] R. Kumaresan and N. Panchal, "Encoding bandpass signals using zero/level crossings: a model-based approach," IEEE Transactions on Audio, Speech, and Language Processing, 2010.

[11] J. Selva, "Functionally weighted Lagrange interpolation of bandlimited signals from nonuniform samples," IEEE Transactions on Signal Processing, vol. 57, no. 1, pp. 168-181, Jan 2009.

[12] E. C. Titchmarsh, "The zeros of certain integral functions," Proc. London Math. Soc., vol. s2-25, pp. 283-302, 1926.

[13] R. Duffin and A. C. Schaeffer, "Some properties of functions of exponential type," Bull. Amer. Math. Soc., vol. 44, no. 4, pp. 236-240, 1938.

[14] Steven M. Kay, Modern spectral estimation, Signal Processing. Prentice Hall, first edition, 1988. 
